\title{
Impact of KRAS, BRAF and microsatellite instability status after cytoreductive surgery and HIPEC in a national cohort of colorectal peritoneal metastasis patients
}

\author{
S. G. Larsen $\mathbb{D}^{1 凶}$, M. A. Goscinski ${ }^{1}$, S. Dueland ${ }^{2}$, S. E. Steigen ${ }^{3}$, E. Hofsli ${ }^{4,5}$, A. Torgunrud ${ }^{5}$, M. Lund-Iversen ${ }^{6}$, V. J. Dagenborg ${ }^{1}$, \\ K. Flatmark ${ }^{1,7}$ and H. Sorbye ${ }^{8}$ \\ (c) The Author(s) 2021
}

\begin{abstract}
BACKGROUND: Patients with metastatic colorectal cancer (mCRC) carrying BRAF (mutBRAF) or KRAS mutation (mutKRAS) have an inferior prognosis after liver or lung surgery, whereas the prognostic role in the context of peritoneal metastasis (PM) after cytoreductive surgery (CRS) and hyperthermic intraperitoneal chemotherapy (HIPEC) has been less investigated.

METHODS: In total, 257 patients with non-appendiceal PM-CRC were included from the Norwegian National Unit for CRS-HIPEC. RESULTS: In total, 180 patients received CRS-HIPEC with Mitomycin C, 77 patients received palliative surgery only. In the CRS-HIPEC group, mutBRAF was found in $24.7 \%$, mutKRAS $33.9 \%$ and double wild-type $41.4 \%$ without differences in survival. MSI was found in $29.3 \%$ of mutBRAF cases. Patients with mutBRAF/MSI had superior 5 -year survival compared to mutBRAF with MSS (58.3\% vs $25.2 \%$, $P=0.022)$, and better 3-year disease-free survival (DFS) compared to mutKRAS (48.6\% vs $17.2 \%, P=0.049)$. Peritoneal Cancer Index and the number of lymph node metastasis were prognostic for OS, and the same two, location and gender prognostic for DFS in multivariate analysis.

CONCLUSIONS: PM-CRC with CRS-HIPEC patients has a surprisingly high proportion of mutBRAF (24.7\%). Survival was similar comparing mutBRAF, mutKRAS and double wild-type cases, whereas a small subgroup with mutBRAF and MSI had better survival. Patients with mutBRAF tumours and limited PM should be considered for CRS-HIPEC.
\end{abstract}

British Journal of Cancer (2022) 126:726-735; https://doi.org/10.1038/s41416-021-01620-6

\section{INTRODUCTION}

Colorectal cancer (CRC) is the third most commonly diagnosed malignancy and the second leading cause of cancer death in the world [1]. Approximately $20 \%$ of patients $[2,3]$ have synchronous metastasis at diagnosis of CRC and $15-25 \%$ of patients develop the metachronous metastatic disease during follow-up $[2,4,5]$. The most frequent metastatic site is the liver (60-74\%), whereas $19-23 \%$ of metastatic CRC ( $\mathrm{mCRC}$ ) patients have peritoneal metastases (PM) [3, 5]. PM-CRC carry a worse prognosis than isolated distant metastases at other sites [6]. Most patients with mCRC cannot be cured, illustrated by a 5-year survival of $10-20 \%$ in study patients $[7,8]$, and an even more grim prognosis in population-based registries with median survival 5-12 months and 5 -year survival of $5-10 \%[9,10]$.

The best chance for long-term survival for patients with mCRC is surgical resection or complete local treatment by any modality. Cytoreductive surgery followed by hyperthermic intraperitoneal chemotherapy (CRS-HIPEC) has shown promising results in patients with limited and resectable PM-CRC. Five-year survival of up to $40 \%$ has been observed in a randomised controlled trial [11], case-control studies [12-14], meta-analysis [15] and cohort studies [16]. Systemic chemotherapy alone has a limited effect on localised PM-CRC with median survival of 13-16 months $[6,17]$. The aim of CRS-HIPEC is to remove all macroscopic tumours and to achieve high intraperitoneal concentrations of hyperthermic cytotoxic drugs [18]. Analysis of BRAF, RAS and microsatellite instability (MSI) status is recommended upfront in patients with $\mathrm{mCRC}$ to tailor systemic treatment. A potentially prognostic value of these markers could be used to aid in the selection of the most optimal patients for CRS-HIPEC. KRAS mutations (mutKRAS) occur in $\sim 40 \%$ of patients with $\mathrm{mCRC}$ and is associated with a worse prognosis after liver [19] and lung surgery [20]. BRAF mutations (mutBRAF) are found in $21 \%$ of unselected population-based patients with $\mathrm{mCRC}[21]$, in $5-11 \%$ of trial patients $[22,23]$ and less

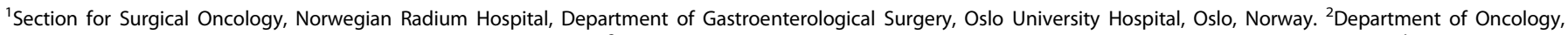

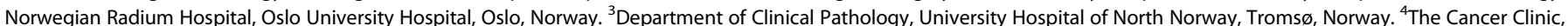

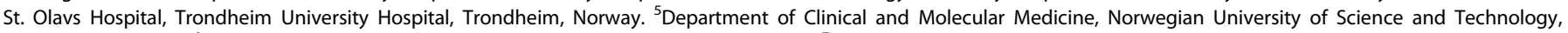

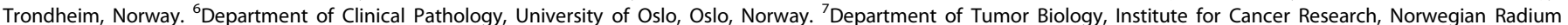

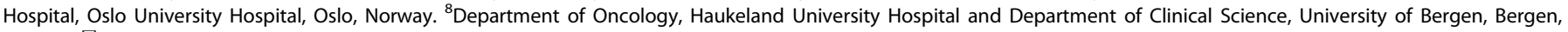
Norway. ${ }^{凶}$ email: stl@ous-hf.no
} 
in patients undergoing liver or lung metastasectomies [21, 24]. $B R A F$ mutations seem to be more frequent in PM-CRC [23]. Several studies have shown a negative prognostic association of $B R A F$ V600E mutations after liver or lung surgery in $\mathrm{MCRC}$ patients $[19,20,23-25]$. mutBRAF status may be a factor to consider when deciding if liver surgery should be offered in patients with very advanced mCRC [26]. MSI is present in 3-8\% in patients with mCRC $[27,28]$. In contrast to Stage II-III disease, MSI carries a worse prognosis in the metastatic setting [29], but is a predictive marker for the benefit of immunotherapy [30]. The relevance of MSI after surgery in MCRC is not known, but may be important as the mismatch repair system has been found important in the interpretation of BRAF mutations in Stage III colon cancer [31].

The possible prognostic role of KRAS and BRAF mutations has not been well studied after CRS-HIPEC in contrast to after liver or lung surgery. A recent Swiss study found that both RAS and BRAF mutations were negative prognostic factors after HIPEC [22] and a Swedish study suggested that mCRC patients with mutBRAF and isolated PM should rather be considered for alternative treatment options than CRS-HIPEC [32]. In a retrospective design, we studied $K R A S, B R A F$ and MSI status in a prospective national cohort from the only national centre for CRS- HIPEC in Norway.

\section{METHODS \\ Patient population}

Between January 2005 and December 2015, 335 patients with PM-CRC were considered for CRS-HIPEC at the Norwegian Radium Hospital, part of Oslo University Hospital. All patients were prospectively registered in the institutional peritoneal surface malignancy database where clinicopathological data, treatment details, and outcome were recorded. Fifty-one patients with appendiceal cancer were excluded from the study. In addition, 27 patients without histologically verified PM at primary surgery or at the time of CRSHIPEC were excluded, resulting in a study population of 257 patients. Missing data were retrospectively collected from patient records from referring hospitals. Information regarding disease recurrence and follow-up was obtained by retrieving patient records and radiologic workup from our outpatients clinic or referring hospitals. The synchronous PM was defined as PM at or within 6 months of primary surgery and disease-free interval (DFI) was the time period from primary surgery to diagnosis of PM. The study was approved by the Norwegian Ethics Committee (s-07160b) and written informed consent was obtained from the patients.

\section{Treatment}

CRS was performed with the intention to remove all macroscopically visible tumours, involving peritonectomy procedures and organ resections as necessary. Peritoneal tumour distribution was classified using the peritoneal cancer index (PCI) and the completeness of cytoreduction (CC) score was used to evaluate residual tumour after CRS. Complete cytoreduction (CC-0) was achieved in $180(70 \%)$ cases and only CC-0 cases were given HIPEC. The remaining 77 cases (30\%) were in a palliative setting, either because of a massive tumour load or extensive small bowel involvement. HIPEC was administrated using the open Coliseum technique until 2008, thereafter a closed technique with an open abdomen was used [33]. The HIPEC regimen contained mitomycin, $35 \mathrm{mg} / \mathrm{m}^{2}$ (maximum $70 \mathrm{mg}$ ), administered for $90 \mathrm{~min}$ in three fractions (50\% initially, $25 \% / 30$ $\mathrm{min}$ and $25 \% / 60 \mathrm{~min})$. Median procedure duration was $420 \mathrm{~min}(180-880)$ and median intraperitoneal temperature during HIPEC $42.0^{\circ} \mathrm{C}$. All anastomoses were completed before the HIPEC procedure. According to Norwegian guidelines, adjuvant chemotherapy was not routinely given. Postoperative complications (30-day morbidity and 100-day mortality) were classified according to Accordion [34].

\section{Histopathology and molecular analysis}

Surgical specimens were collected and fixed in $4 \%$ buffered formaldehyde and subsequently embedded in paraffin followed by routine histological investigation on 3-4- $\mu \mathrm{m}$-thin haematoxylin-eosin-stained slides. In 18 cases, tumour tissue was frozen in liquid nitrogen immediately after resection and stored at $-80^{\circ} \mathrm{C}$ in a tissue bank.

All cases with unavailable or unknown mutational status were retrospectively collected and reviewed by a pathologist ( 100 cases). DNA eluat from previous ancillary tests was used if available. If unavailable, DNA was extracted from representative tumour areas using a Qiagen kit (Hilden, Germany). DNA quality was measured with Nanodrop ${ }^{\mathrm{TM}}$ (ThermoFisher, Waltham, Massachusetts, U.S.) and analysed for mutKRAS in exons 2, 3 and 4 (KRAS Mutation Analysis, Entrogen) and for BRAF (V600E/K/D mutations) investigated with allele-specific real-time PCR (in-house setup, protocol available on request). KRAS exon 2, 3 and 4 were performed or available in all cases, and when KRAS was wild type we added BRAF testing. NRAS was not tested in all KRAS wild-type cases and left out of the study for the reason that expanding RAS analyses have had very little impact on results as shown in the Nordic 7 study [35]. MSI status was determined with PCR analysis on customised molecular MSI panels with the following markers: (BAT 26 (HMSH2 intron), BAT25 (c-KIT intron), NR24 (Zinc finger 2, 3'UTR), NR21 (SLC7A8, 5'UTR), TGF-Beta-RII (c.374-3c383), BAT 40 (1p13.1), CAT25 (CASP2,3'UTR), RCC2 (5'UTR). Changes in $3 / 8$ markers were defined as microsatellite instable phenotype. As a control, a general microsatellite stable (MSS) DNA sample was used (in-house setup, protocol available on request). The analysis was performed from the primary tumour in all synchronous cases (98) and from primary (45) or metastatic tumours (100) in metachronous cases. Most analyses have been performed in the last 3-5 years.

In analyses regarding the CRS-HIPEC group $(n=174)$, the 57 patients with palliative or explorative operations were excluded as well as 4 patients with missing tumour blocks and 1 with unsuccessful genetic analysis. One patient was lost on follow-up. When frozen tumour tissue samples were used, they were homogenised and disrupted using TissueLyzer LT from Qiagen (Hilden, Germany). DNA was then extracted from the lysate using the AllPrep DNA/RNA/miRNA Universal Kit (Qiagen, Hilden, Germany). DNA concentrations and purity were evaluated using ThermoFisher NanoDrop spectrophotometer, and the $A_{b s_{260 / 280}}>1.8$ for all the samples. Targeted DNA sequencing was performed using the lon Torrent PGM Personal Genome Machine and the Ion AmpliSeqTM Cancer Hotspot Panel v2 (ThermoFisher Scientific, Waltham, MA, USA), covering $\sim 2800$ hotspot mutations in 50 cancer-related genes. The Torrent Suite Variant Caller, with the manufacturer's recommended settings, was used to generate single nucleotide variants and small insertions/deletions with a variant allele frequency threshold of two percent. The sequencing depth exceeded $500 \times$ for $98 \%$ of all amplicons (median depth of $>4000 \times$ ). Every detected mutation was manually reassessed using Integrative Genomics Viewer and functionally annotated with ANNOVAR [36], using RefSeq as the underlying gene model and information from the 1000 Genomes Project (1000genomes.org) and the Catalogue of Somatic Mutations in Cancer (cancer.sanger.ac.uk/cosmic).

\section{Statistical analysis}

Categorical variables were described using frequencies/percentages and continuous variables were described with median/range. Associations between clinicopathological parameters and the extent of surgery were analysed using chi-squared tests (Pearson's or linear-by-linear association). Continuous variables were analysed using Kruskal-Wallis tests. Univariate analysis was performed using the Kaplan-Meier method. Survival data were obtained from the Norwegian Cause of Death Registry and patients alive on November 1, 2017 were censored. Time from PM surgery to death or censoring date in the analyses of OS and to time of peritoneal relapse, distant metastasis, death or last follow-up in analyses of disease-free survival (DFS) were used. The log-rank test was used to compare differences in survival. Factors significant in univariate analysis for OS (mutational status, $\mathrm{PCl}$, number of lymph node metastasis) and for DFS (right or left-sided tumour in addition) were further examined using the multivariable Cox proportional hazards regression model (enter), as well as age and gender. The number of variables is restricted to 5 in OS analysis and 6 in DFS analyses of the 167 cases and therefore no corrections are applied. mutBRAF with MSS/ mutBRAF with MSI/ mutKRAS/ double wild type (double wt) were tested together in the multivariate analysis because they were mutually exclusive. Statistical analyses were conducted using SPSS software (version 25.0, SPSS Inc, IL, USA). $P<0.05$ were considered statistically significant.

\section{RESULTS}

\section{Clinical variables and histopathology}

In total, 174 patients received CRS-HIPEC (treatment group) and had a median $\mathrm{PCl}$ of 9 , whereas the 77 patients in the palliative group had median $\mathrm{PCl}$ of 29 . Table 1 summarises the clinicopathological characteristics of the study cohort. The palliative 
Table 1. Characteristics of metastatic colorectal cancer patients with radical treatment (CRS-HIPEC, $n=180$ ) or palliative/ explorative treatment $(n=77)$.

\begin{tabular}{|c|c|c|c|c|c|}
\hline \multirow[t]{2}{*}{ Parameter } & \multicolumn{2}{|c|}{$\begin{array}{l}\text { CRS-HIPEC } \\
(n=180)\end{array}$} & \multicolumn{2}{|c|}{$\begin{array}{l}\text { Palliative } \\
\text { treatment } \\
(n=77)\end{array}$} & \multirow[t]{2}{*}{$P$} \\
\hline & $n$ & $\%$ & $n$ & $\%$ & \\
\hline Gender & & & & & 0.095 \\
\hline Female & 115 & 63.9 & 40 & 51.9 & \\
\hline Male & 65 & 36.1 & 37 & 48.1 & \\
\hline $\begin{array}{l}\text { Age, median } \\
\text { (year, range) }\end{array}$ & 59 & $22-77$ & 58 & $20-72$ & 0.313 \\
\hline T-stage & & & & & $<0.001$ \\
\hline T1-2 & 1 & 0.6 & 2 & 2.9 & \\
\hline T3 & 74 & 44.0 & 13 & 18.8 & \\
\hline T4 & 93 & 55.4 & 54 & 78.3 & \\
\hline Not reported & 12 & & 8 & & \\
\hline $\mathrm{N}$-stage & & & & & 0.315 \\
\hline No & 51 & 28.7 & 17 & 24.3 & \\
\hline N1 & 60 & 33.7 & 19 & 27.1 & \\
\hline N2 & 67 & 37.6 & 34 & 48.6 & \\
\hline Not reported & 2 & & 0 & & \\
\hline $\begin{array}{l}\text { Number of metastatic } \\
\text { lymph nodes, median } \\
\text { (range) }\end{array}$ & 2 & $0-34$ & 3 & $0-28$ & 0.125 \\
\hline $\begin{array}{l}\text { Grade of tumour } \\
\text { differentiation }\end{array}$ & & & & & 0.615 \\
\hline Poorly & 47 & 31.8 & 19 & 38.0 & \\
\hline Moderate & 94 & 63.5 & 28 & 56.0 & \\
\hline Well & 7 & 4.7 & 3 & 6.0 & \\
\hline Not reported & 32 & & 27 & & \\
\hline Signet ring cells & & & & & 0.023 \\
\hline Present & 15 & 12.5 & 9 & 31.0 & \\
\hline Absent & 105 & 87.5 & 20 & 69.0 & \\
\hline Not reported & 60 & - & 48 & - & \\
\hline Tumour location 1 & & & & & 0.017 \\
\hline Right colon & 76 & 42.2 & 45 & 58.4 & \\
\hline $\begin{array}{l}\text { Left colon } \\
\text { and rectum }\end{array}$ & 104 & 57.8 & 32 & 41.6 & \\
\hline Tumour location 2 & & & & & 0.367 \\
\hline Colon & 162 & 90.0 & 72 & 93.5 & \\
\hline Rectum & 18 & 10.0 & 5 & 6.5 & \\
\hline \multicolumn{6}{|l|}{ Peritoneal metastases } \\
\hline Synchronous & 61 & 33.9 & 37 & 48.1 & 0.036 \\
\hline Metachronous & 119 & 66.1 & 40 & 51.9 & \\
\hline $\begin{array}{l}\text { Chemoterapy earlier } \\
\text { than CRS-HIPEC }\end{array}$ & & & & & 0.002 \\
\hline Yes & 124 & 68.9 & 40 & 51.9 & \\
\hline No & 56 & 31.1 & 37 & 48.1 & \\
\hline ASA & & & & & 0.011 \\
\hline 1 & 2 & 1.7 & 3 & 5.3 & \\
\hline 2 & 108 & 92.3 & 43 & 75.4 & \\
\hline 3 & 7 & 6.0 & 11 & 19.3 & \\
\hline Not reported & 63 & & 20 & & \\
\hline $\begin{array}{l}\text { CEA (median, } \\
\text { range) }(\mu \mathrm{g} / \mathrm{L})\end{array}$ & 4 & $1-1820$ & 6 & $1-2562$ & 0.224 \\
\hline
\end{tabular}

Table 1 continued

\begin{tabular}{|c|c|c|c|c|c|}
\hline \multirow[t]{2}{*}{ Parameter } & \multicolumn{2}{|c|}{$\begin{array}{l}\text { CRS-HIPEC } \\
(n=180)\end{array}$} & \multicolumn{2}{|c|}{$\begin{array}{l}\text { Palliative } \\
\text { treatment } \\
(n=77)\end{array}$} & \multirow[t]{2}{*}{$P$} \\
\hline & $n$ & $\%$ & $n$ & $\%$ & \\
\hline $\begin{array}{l}\text { CA } 19-9 \text { (median, } \\
\text { range) (U/L) }\end{array}$ & 18.5 & $5-1175$ & 32 & $0-764$ & 0.232 \\
\hline $\mathrm{PCl}$ & & & & & $<0.001$ \\
\hline $0-10$ & 111 & 61.7 & 6 & 7.8 & \\
\hline $11-20$ & 58 & 32.2 & 7 & 9.1 & \\
\hline $21-30$ & 10 & 5.6 & 37 & 48.1 & \\
\hline$>30$ & 1 & 0.6 & 27 & 35.1 & \\
\hline $\mathrm{PCl}$, median (range) & 9 & $0-28$ & 29 & $2-39$ & $<0.001$ \\
\hline Mutational status & & & & & 0.530 \\
\hline Double wt & 72 & 41.1 & 32 & 43.8 & \\
\hline mut $B R A F$ & 43 & 24.6 & 13 & 17.8 & \\
\hline mutKRAS & 60 & 34.3 & 28 & 38.4 & \\
\hline Missing & 5 & - & 4 & - & \\
\hline BRAF & & & & & 0.430 \\
\hline mut $B R A F$ with MSS & 29 & 16.8 & 13 & 17.8 & \\
\hline mutBRAF with MSI & 12 & 6.9 & 2 & 2.7 & \\
\hline wtBRAF & 132 & 76.3 & 58 & 79.5 & \\
\hline Missing & 7 & - & 4 & - & \\
\hline MSS/MSI & & & & & 0.849 \\
\hline MSS & 96 & 86.5 & 43 & 91.5 & \\
\hline MSI & 15 & 13.5 & 4 & 8.5 & \\
\hline Not analysed & 69 & - & 30 & - & \\
\hline Complications & & & & & 0.064 \\
\hline Accordion $0-2$ & 135 & 75.0 & 72 & 93.5 & \\
\hline Accordion 3-6 & 45 & 25.0 & 5 & 6.5 & \\
\hline $\begin{array}{l}\text { Hospital stay (median } \\
\text { days, range) }\end{array}$ & 10 & $5-57$ & 7 & $2-24$ & $<0.001$ \\
\hline $\begin{array}{l}\text { Operation time } \\
\text { (median } \\
\text { minutes, range) }\end{array}$ & 420 & $180-880$ & 150 & $30-485$ & $<0.001$ \\
\hline
\end{tabular}

group differed from the treatment group regarding the following parameters: more ASA 3 patients, worse T-stage, more right-sided tumours and more specification of signet ring cells in the tumours, more synchronous disease, more systemic chemotherapy and higher PCl-index. In the treatment group, 45 patients $(25.0 \%)$ had Accordion groups 3-5 complications and there was no 100-day mortality. In the palliative group, $6.5 \%$ had Accordion groups 3-5 complications and there were no deaths within 30 days.

\section{Molecular analysis}

We analysed tumour tissue and performed DNA analysis for mutations in KRAS, BRAF and analyses for MSS/MSI in all patients except 8 of 257 (3.1\%) where tumour tissue was not obtained. There was no significant difference in the frequency between mutKRAS, mutBRAF or double wt between CRS-HIPEC and palliative groups.

Table 2 shows mutation analyses in the 174 CRS-HIPEC patients; mutKRAS ( $n=59,33.9 \%)$, mutBRAF $(n=43,24.7 \%)$ and double wt ( $n=72,41.4 \%)$. There were significant differences regarding primary tumour location, tumour differentiation and CEA. More mutBRAF were found in the right colon, whereas no mutBRAF rectal cancer cases were found (Table 2). There were more cases with elevated CEA values in the mutKRAS group (61.0\%) than in 
Table 2. Tumour mutation analysis (KRAS/BRAF) in metastatic colorectal cancer patients with cytoreductive surgery and HIPEC (CRS-HIPEC) $(n=174)$.

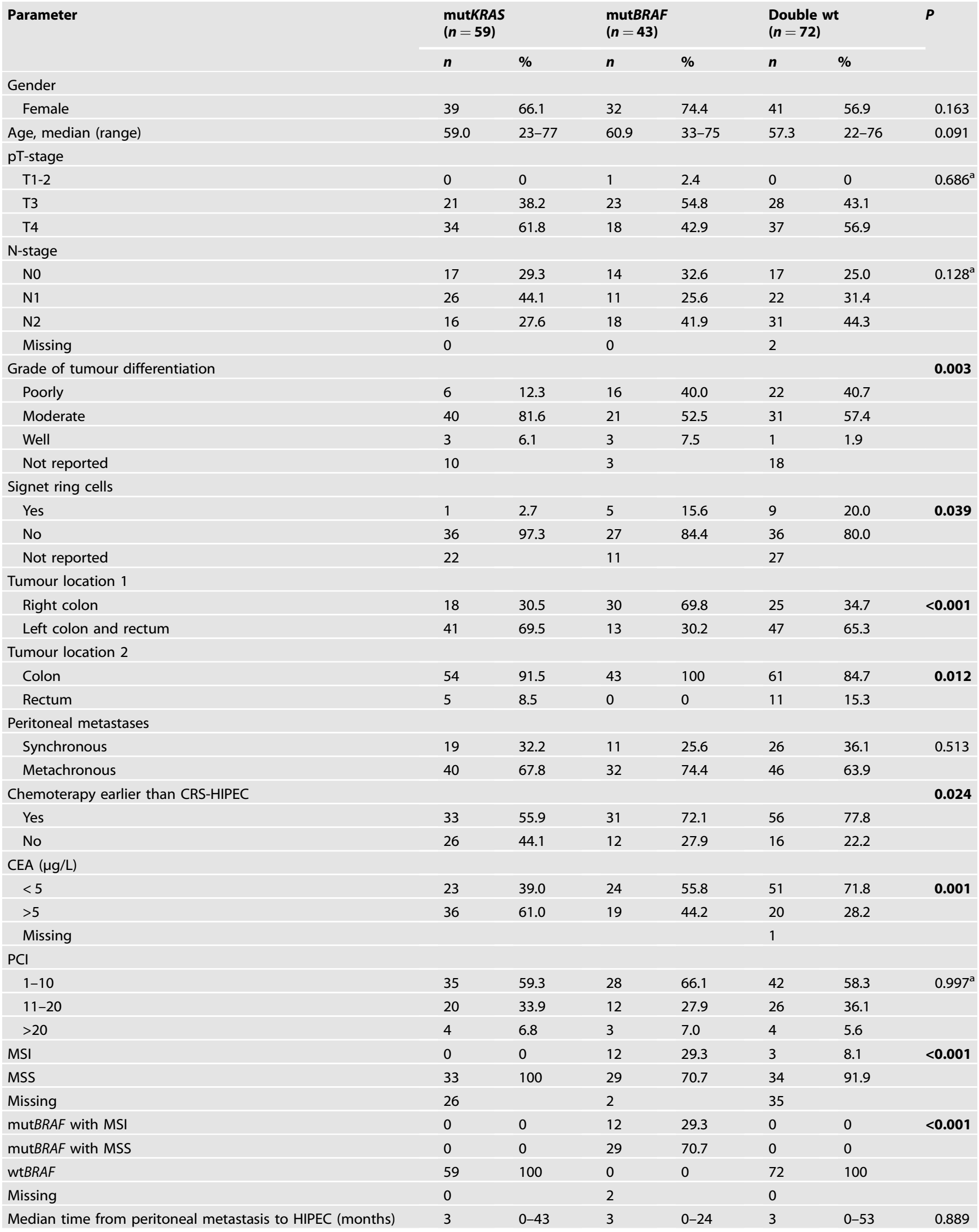


Table 2 continued

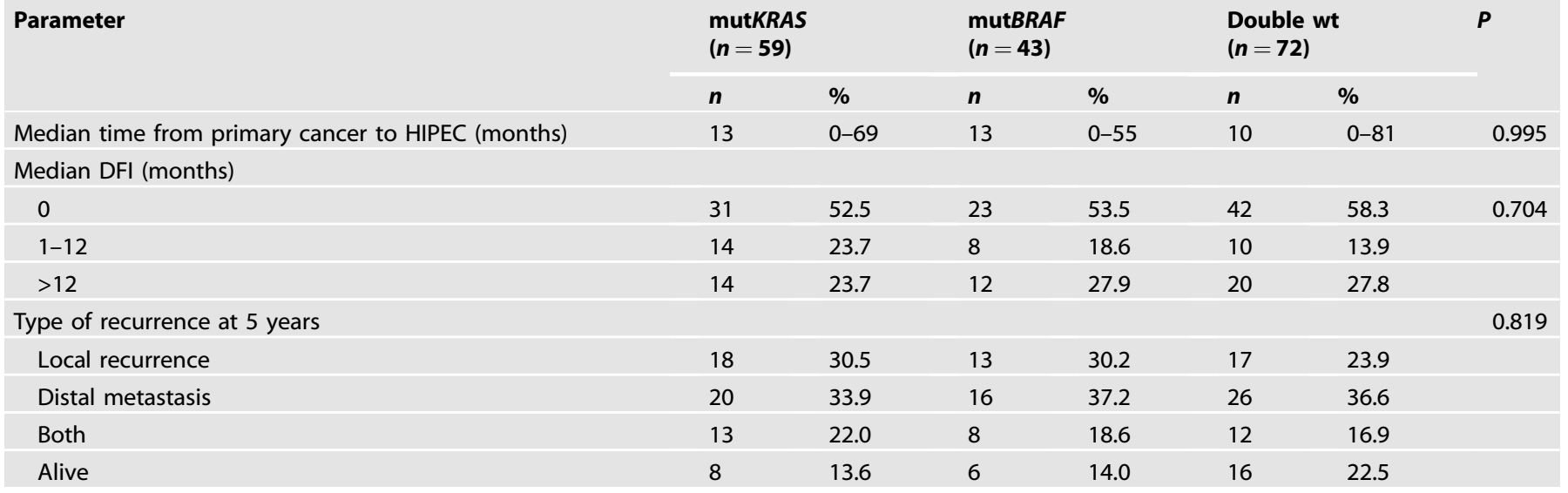

aLinear-by-linear association.

Statistically significant $p<0.05$ values are in bold.
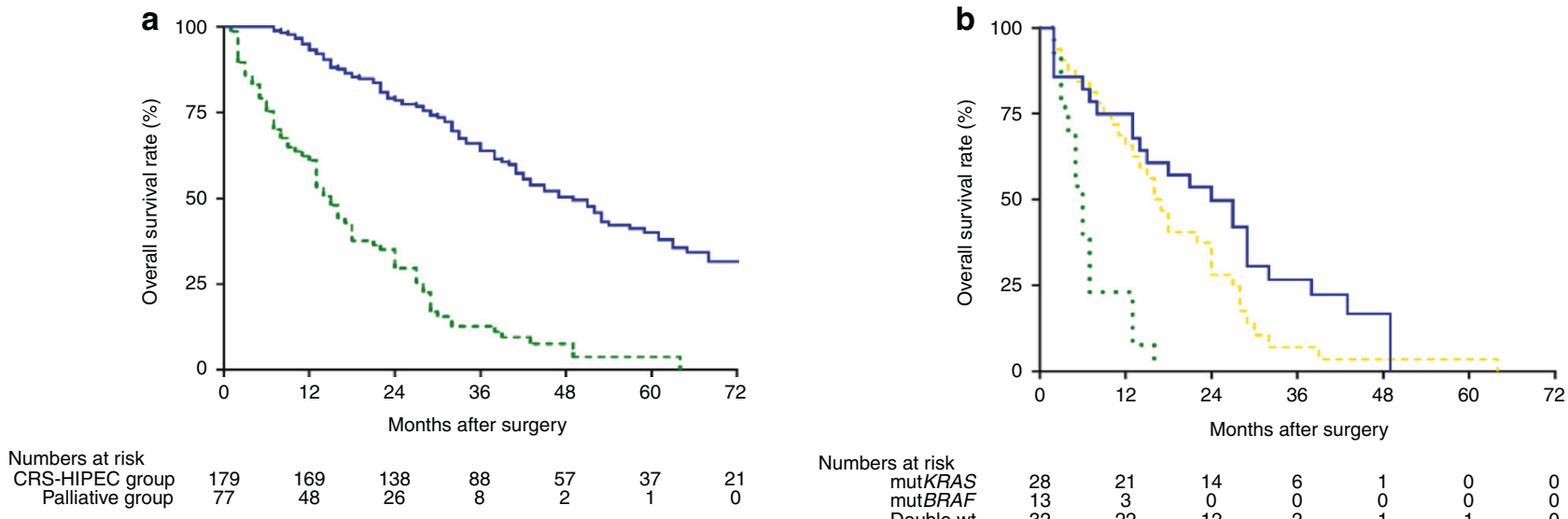

$\begin{array}{cccccccc}\text { RS-HIPEC group } & 179 & 169 & 138 & 88 & 57 & 37 & 21 \\ \text { Palliative group } & 77 & 48 & 26 & 8 & 2 & 1 & 0\end{array}$
Numbers at risk
mutKRAS

$\begin{array}{lcccccc}28 & 21 & 14 & 6 & 1 & 0 & 0 \\ 13 & 3 & 0 & 0 & 0 & 0 & 0 \\ 32 & 22 & 12 & 2 & 1 & 1 & 0\end{array}$

Fig. 1 Kaplan-Meier plot showing time from surgery on the $x$ axis and estimated overall survival on the $y$ axis. a Overall survival of CRSHIPEC versus palliative surgery. The blue line represents the CRS-HIPEC group and the green dashed line represents the patients in the palliative group. Log-rank test shows a significant difference between the two groups with $P<0.001$. b Overall survival comparing mutation status after palliative resection. Kaplan-Meier plot with time from surgery on the $x$ axis and estimated overall survival on the $y$ axis. The blue line represents the KRAS-mutated tumours (mutKRAS) tumours and the green dotted line represents the BRAF-mutated (mutBRAF) tumours. The gold dashed line represents the patients with KRAS and BRAF wild-type (double wt) tumours. Log-rank test shows a significant difference between the three groups with $P<0.001$.

Table 3. Survival of metastatic colorectal cancer patients after cytoreductive surgery and HIPEC according to mutational status in univariate analysis $(n=174)$.

\begin{tabular}{|c|c|c|c|c|c|c|}
\hline & $\begin{array}{l}\text { All HIPEC } \\
(n=179)\end{array}$ & $\begin{array}{l}\text { mutKRAS } \\
(n=59)\end{array}$ & mutBRAF total [43] & $\begin{array}{l}\text { Double wt } \\
(n=72)\end{array}$ & $\begin{array}{l}\text { mutBRAF with MSS } \\
(n=29)\end{array}$ & $\begin{array}{l}\text { mutBRAF with MSI } \\
(n=12)\end{array}$ \\
\hline Median DFS (mnt, 95\% Cl) & $11(9.2-12.8)$ & $11(7.8-14.2)$ & $11(7.3-14.7)$ & $10(7.5-12.5)$ & $10(8.7-11.3)$ & $35(11.1-58.9)$ \\
\hline 3-year DFS (\%) & 19.9 & 13.2 & 22.4 & 23.8 & 17.2 & 48.6 \\
\hline Median OS (mnt, $95 \% \mathrm{Cl}$ ) & $49(41.7-56.3)$ & $47(35.2-58.8)$ & $51(37.7-64.3)$ & $45(33.3-56.7)$ & $42(27.1-56.9)$ & Not reached \\
\hline 5-year OS (\%) & 40.1 & 42.1 & 35.6 & 40.8 & 25.2 & 58.3 \\
\hline
\end{tabular}

mutBRAF $(44.2 \%)$ and double wt $(28.2 \%, P<0.001)$. No association was seen between $\mathrm{PCl}$ level and mutational status (Table 2). All cases with mutBRAF and half of the other cases were tested for microsatellite instability (MSI). In all, 29 mutBRAF patients $(70.7 \%)$ were MSS and 12 mutBRAF patients (29.3\%) MSI. In mutBRAF tumours, MSI were more often diagnosed in cases with synchronous PM-CRC ( $50 \%$ vs $17.9 \%, P=0.047)$, and with poorly differentiated tumours $(83.8 \%$ vs $15.4 \%, P<0.001)$.

\section{Survival}

Median OS was 49 months after CRS-HIPEC in contrast to 15 months after laparotomy for the palliative group $(P<0.001)$, 5 -year survival rates were $40.1 \%$ vs $3.8 \%$ (Fig. 1 a and Table 3 ). Median DFS after CRS-HIPEC was 11 months (not shown). In the palliative group, patients with mutBRAF had a worse median survival (6 months) compared to patients with mutKRAS (24 months, $P<0.001)$ or double wt (16 months, $P<0.001$, 

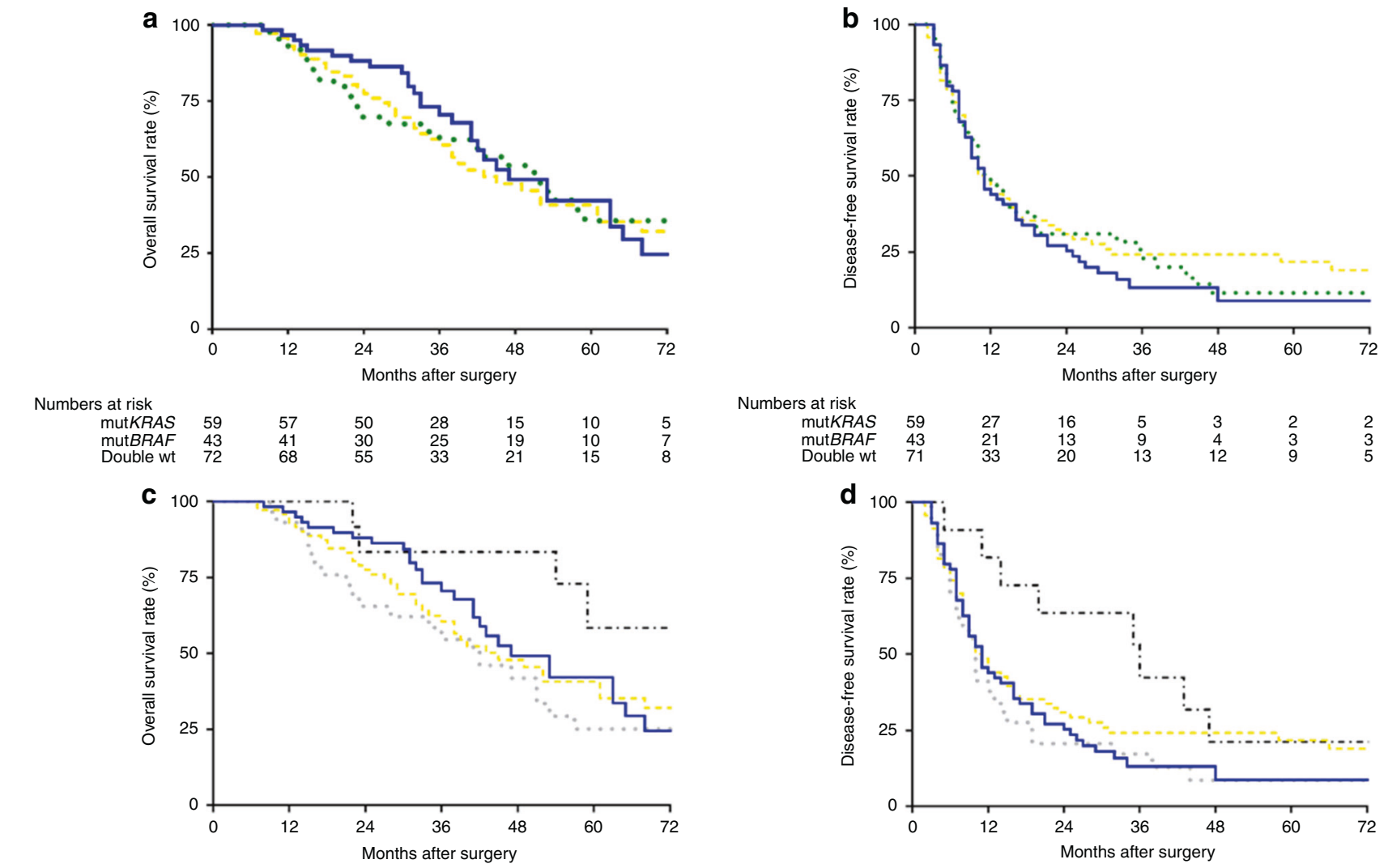

Numbers at risk
mutKRAS
mutBRAF/MSS
Double wt
mutBRAF/MSI

$\begin{array}{lllllll}59 & 57 & 50 & 28 & 15 & 10 & 5 \\ 29 & 27 & 19 & 15 & 10 & 6 & 4 \\ 72 & 68 & 55 & 33 & 21 & 15 & 8 \\ 12 & 12 & 10 & 9 & 8 & 4 & 3\end{array}$
Numbers at risk
mutKRAS
mutBRAF/MSS
Double wt
Double wt

$\begin{array}{ll}59 & 27 \\ 29 & 12 \\ 71 & 33 \\ 12 & 9\end{array}$

$\begin{array}{ccc}27 & 16 & \\ 12 & 6 & 4 \\ 33 & 20 & 13 \\ 9 & 7 & 5\end{array}$

5
4
13
5

$\begin{array}{ccc}3 & 2 & 2 \\ 2 & 2 & 2 \\ 12 & 9 & 5 \\ 2 & 1 & 1\end{array}$

Fig. 2 Kaplan-Meier plot with time from surgery on the $x$ axis and estimated overall survival or disease free survival on the $y$ axis. a Overall survival after CRS-HIPEC based on mutation status. The blue line represents the KRAS-mutated tumours (mutKRAS) tumours and the green dotted line represents the BRAF-mutated (mutBRAF) tumours. The gold dashed line represents the patients with KRAS and BRAF wildtype (double wt) tumours. Log-rank test shows significant difference between mutBRAF vs mutKRAS, $P=0.046$ and between mutBRAF vs double wt, $P<0.001$. b Disease-free survival after CRS-HIPEC based on mutation status. Kaplan-Meier plot with time from surgery on the $x$ axis and estimated overall survival on the $y$ axis. The blue line represents the KRAS-mutated tumours (mutKRAS) and the green dotted line represents the BRAF-mutated (mutBRAF) tumours. The gold dashed line represents the patients with KRAS and BRAF wild-type (double wt) tumours. Log-rank test is ns. c Overall survival comparing mutation and microsatellite instability (MSI) status. Kaplan-Meier plot with time from surgery on the $x$ axis and estimated overall survival on the $y$ axis. The blue line represents the KRAS-mutated tumours (mutKRAS) tumours and the green dotted line represents the BRAF-mutated microsatellite stable (MSS) (mutBRAF/MSS) tumours. The gold dashed line represents the patients with KRAS and BRAF wild-type (double wt) tumours and the black dashed/dotted line represents the BRAF-mutated microsatellite instable (MSI (mutBRAF/MSI) tumours. Log-rank test shows a significant difference between mutBRAF groups with MSI or MSS with $P=0.022$. d Disease-free survival after CRS-HIPEC based on mutation and microsatellite instability (MSI) status. Kaplan-Meier plot with time from surgery on the $x$ axis and estimated overall survival on the $y$ axis. The blue line represents the KRAS-mutated tumours (mutKRAS) tumours and the grey dotted line represents the BRAF-mutated microsatellite stable (MSS) (mutBRAF/MSS) tumours. The gold dashed line represents the patients with KRAS and BRAF wild-type (double wt) tumours and the black dashed/dotted line represents the BRAF-mutated microsatellite instable (MSI) (mutBRAF/MSI) tumours. Log-rank test shows a significant difference between mutBRAF/MSI group and mutKRAS group with $P=0.049$.

Fig. 1b). There was no significant difference between OS and DFS after CRS-HIPEC when stratifying for mutKRAS, mutBRAF or double wt (Fig. 2a, b). However, CRS-HIPEC patients with mutBRAF and MSS had shorter median OS (42 months) than those with mutBRAF and MSI where median survival was not reached in the study period and the corresponding 5-year OS rates were $25.2 \%$ vs $58.3 \%$ (Fig. 2 c $P=0.022$ ). Patients with mutBRAF and MSI also had a superior DFS compared to mutKRAS patients (Fig. $2 \mathrm{~d}, P=0.049$ ). There was no association between mutation status and type of recurrence (Table 2). PCI (HR 1.084) and the number of lymph node metastasis in the primary tumour (HR 1.056) were predictors of OS in the multivariate analysis, for every increase in $\mathrm{PCl}$ value or for the increase in the number of metastatic lymph nodes. Lymph node metastasis, $\mathrm{PCl}$, tumour location and gender were all predictors for DFS (Table 4).

\section{DISCUSSION}

In this national cohort of PM-CRC patients treated with CRS-HIPEC, we found a high incidence of BRAF mutations (24\%), and in contrast to two prior reports, we did not see any differences in survival after CRS-HIPEC according to KRAS or BRAF mutational status. Patients with mutBRAF and MSI had significantly better survival than all other groups. Our results suggest that $\mathrm{mCRC}$ patients with limited PM and mutBRAF should be considered for CRS-HIPEC.

\section{Mutations and site of metastasis}

In patients with $\mathrm{mCRC}$, an incidence of $35-40 \%$ mutKRAS and 5-20\% BRAF mutations are usually observed [20, 21, 37]. However, the mutations seem to be associated with a distinct pattern of metastatic spread. The presence of a KRAS mutation is associated with a lower frequency of liver metastases and a higher frequency 
Table 4. Multivariable Cox regression analysis of OS and DFS after CRS-HIPEC in patients with PM-CRC $(n=167)$.

\begin{tabular}{|c|c|c|c|c|c|c|}
\hline \multirow[b]{2}{*}{ Variable } & \multirow{2}{*}{$\begin{array}{l}\text { OS } \\
\text { HR }\end{array}$} & \multicolumn{5}{|c|}{ DFS } \\
\hline & & $(95 \% \mathrm{Cl})$ & $\boldsymbol{P}$ & HR & $(95 \% \mathrm{Cl})$ & $P$ \\
\hline $\mathrm{PCl}$ & 1.084 & $1.05-1.12$ & $<0.001$ & 1.081 & $1.05-1.06$ & $<0.001$ \\
\hline Primary tumour localisation*(ref left colon/rectum) & - & - & - & 0.684 & $0.47-0.98$ & 0.048 \\
\hline Gender (ref male) & 0.843 & $0.55-1.30$ & 0.437 & 0.698 & $0.49-0.99$ & 0.046 \\
\hline Age & 0.995 & $0.98-1.01$ & 0.604 & 1.001 & $0.98-1.02$ & 0.906 \\
\hline mutBRAF with MSI (ref) & & & 0.283 & & & 0.512 \\
\hline mutKRAS & 2.017 & $0.69-5.86$ & 0.197 & 1.496 & $0.71-3.16$ & 0.291 \\
\hline mut $B R A F$ with MSS & 2.83 & $0.94-8.52$ & 0.064 & 1.591 & $0.72-3.50$ & 0.249 \\
\hline Double wt & 2.23 & $0.77-6.43$ & 0.137 & 1.231 & $0.59-2.57$ & 0.580 \\
\hline
\end{tabular}

OS overall survival, DFS disease-free survival, CRS cytoreductive surgery, HIPEC hypertherm intraperitoneal chemotherapy, $P M$ peritoneal metastasis, CRC colorectal cancer, $P C I$ peritoneal cancer index, MSI microsatellite instable tumour, MSS microsatellite stable tumour, ${ }^{*}$ tumour localisation (right colon vs left colon/rectum (ref), ref reference.

of lung, brain and bone metastases [38]. Patients with tumour $B R A F$ mutations are less likely to present with liver limited metastasis $(41 \%$ vs $63 \%)$, but these mutations are more often associated with peritoneal involvement (26\% vs $14 \%$ ) [23]. The mutational pattern is however different in $\mathrm{mCRC}$ patients receiving surgery for metastasis. In liver resected patients, mutKRAS are seen in $28-52 \%$ of cases [24, 39], whereas BRAF mutations are only in $2-5 \%$ of cases $[24,26]$. In the lung, resected patients' mutKRAS is found in $48-62 \%$ of cases $[20,40]$ and BRAF mutations in $0-10 \%$ of cases $[20,40]$. In mCRC patients treated with CRS-HIPEC, mutKRAS are reported in $42-58 \%$ of cases [19, 41]. In the far largest published study by Schneider et al. on 494 patients with CRS-HIPEC, $38 \%$ had KRAS mutation and only $5.8 \%$ $B R A F$ mutation [22]. These results are in contrast to our results where we found a higher mutBRAF rate of $24.7 \%$ among our 174 CRS-HIPEC patients, and $26 \%$ by Yaeger [23]. The reason for this large difference is difficult to explain. However, BRAF mutations are more frequently seen in population-based cohorts compared to phase III studies and reports from tertiary referral centres [21]. The present cohort represents PM from all Norwegian patients accepted for CRS-HIPEC treatment, and thereby more accurately reflects the general population. Our results are relatively similar to Franko et al. who found $12 \%$ BRAF mutations in patients with multifocal mCRC including peritoneal involvement, but $18 \%$ if the patient had isolated peritoneal involvement [6].

\section{Mutations and CRS-HIPEC}

In the evaluation of patients for resection of metastatic disease, resection of all metastatic lesions is the primary objective. However, rapid recurrence in many patients is a major challenge in the treatment of $\mathrm{mCRC}$ patients. Known risk factors associated with poor outcome after surgery may help to select appropriate cases for surgery. At present, the well-known factors for prognosis after CRS-HIPEC are the level of $\mathrm{PCl}[19,42]$, lymph node metastasis ( $\mathrm{N}+$ disease) [38], completeness of cytoreduction [38] and presence of signet ring cell differentiation [38-40]. In our study, PCl level (0-10 vs 10-20) and lymph node status did not vary according to mutational status, whereas signet ring cell differentiation was less frequent in cases with KRAS mutations.

In our study, median survival was 49 months from the time of the CRS-HIPEC and the estimated 5-year survival was $40.1 \%$ which is in concordance with results from other tertiary referral centres [15]. CRS-HIPEC is often performed some months after diagnosing PM due to recent surgery or systemic chemotherapy. When estimating survival from the first verification of $P M$, the median survival time for both radical and palliative treatment increases to 57 months and 20 months $(P<0.001)$ as well as the 5 -year survival rates to $49.0 \%$ and $6.7 \%$.

The use of systemic chemotherapy in CRS-HIPEC can either be given sporadically, as formal adjuvant or neoadjuvant treatment or routinely as in the PRODIGE 7 trial were nearly all cases were pretreated with six or more cycles thereby possibly selecting a population with favourable tumour biology before CRS-HIPEC. In Norway, systemic chemotherapy is routinely given in adjuvant settings to patients with $\mathrm{N}+$ disease, whereas neoadjuvant treatment only to selected cases with extensive PM. None of the cases in this study has received immunotherapy as this treatment was first approved in Norway in September 2019 for MSI mCRC cases.

\section{Mutations and survival in $\mathrm{MCRC}$}

In recent years, knowledge of the tumour-related genomic alterations has led to more precision-based management of patients with $\mathrm{mCRC}$, both with regards to prognostic value and prediction of tumour response to systemic treatment. mutBRAF patients are less likely to undergo metastasectomy ( $26 \%$ vs $41 \%$ ) [23] due to the increased risk of recurrence and worse prognosis [24-26], especially in patients with MSS tumours [43]. MSI is present in about $15 \%$ of patients with localised disease and $7 \%$ in patients with mCRC [29]. mCRC tumours with MSI are more often $B R A F$ mutated compared to MSS $\mathrm{mCRC}(87 \%$ vs. $16 \%)$, and $\mathrm{mCRC}$ patients with MSI receive less often secondary surgery [29]. Survival rates after radical surgery for $\mathrm{MCRC}$ varies according to mutation status. KRAS mutation and especially mutBRAF are negative prognostic factors after liver surgery [23, 24, 44]. After hepatectomy, 5 -year survival was $37 \%$ in mutBRAF vs $67 \%$ for wtBRAF [26] and median survival was inferior in mutBRAF (23 months) compared to 42 months in mutRAS and 63 months in double wt in another study [45]. Several authors suggest that $B R A F$ status should be taken into consideration prior to liver surgery in patients with extensive liver disease [45]. However, a recent case-matched controlled study showed that mutBRAF is not associated with an increased risk of relapse after liver resection for $\mathrm{mCRC}$, thereby supporting considering surgical treatment for resectable liver metastasis in mutBRAF patients [46]. Five-year survival after lung surgery in $\mathrm{mCRC}$ patients was $0 \%$ for mutBRAF, $44 \%$ for mutKRAS and $100 \%$ for double wt [20] with corresponding median survival rates of 15 months, 55 months and 98 months respectively. This gave rise to the question if BRAF-mutated patients should be excluded from lung surgery [20]. 


\section{Mutations and survival after CRS-HIPEC}

Survival after CRS-HIPEC was first reported in a large study where Schneider et al found that both KRAS and BRAF mutations had a worse median cancer-specific survival: 18 months for mutBRAF, 38 months for mutKRAS compared to 52 months for double-wt patients [22]. In another study on 152 patients with CRS, results from next-generation sequencing technology were available for 68 cases: BRAF mutations (6.6\%), but not mutKRAS (46.7\%) were associated with worse survival [47]. Graf et al. found that $B R A F$ mutations among 111 patients with PM-CRC were an independent negative prognostic marker for survival, but not KRAS [32]. The authors suggest that patients with $B R A F$ mutations should be considered for alternative treatment options rather than CRSHIPEC. The results from the above studies are in major contrast to our results where patients with mutBRAF had the same OS and DFS as mutKRAS and double-wt tumours. The reason for this discrepancy may be due to several factors. The study of Graf et al included appendiceal primaries and all cases considered for CRSHIPEC including also palliative cases. This is in contrast to our results where patients receiving CRS-HIPEC had primary tumours located in the colon and rectum only, and not appendix, and in our study we also separated between HIPEC cases and palliative cases not receiving curative surgery in the end. In our palliative cases without CRS-HIPEC, BRAFmut was a poor prognostic factor. In the study of Schneider et al., only $5.8 \%$ of cases were mutBRAF $(22 / 378)$, only $1 / 4$ th of the frequency of $24 \%$ mutBRAF in our study $(43 / 180)$. This could be due to their function as a tertiary referral centre, which generally sees less mutBRAF mCRC cases than seen in the general population [21]. Data from previous publications suggest a high degree of heterogeneity in the outcome of PM-CRC patients with mutBRAF $[48,49]$. In another study on PM-CRC patients treated with HIPEC, mutKRAS was not associated with survival [32].

\section{MSI and CRS-HIPEC}

Studies of primary CRC have shown that mismatch repair status is important in their interpretation of BRAF mutations status, and that mutBRAF does not affect OS and DFS in patients with MSI tumours $[50,51]$. CRC patients with MSI have less recurrence and better survival after radical surgery in Stage II-III disease [43], whereas in $\mathrm{mCRC}$ both $\mathrm{MSI}$ and mutBRAF are independent negative prognostic factors [52]. Sherman et al. found that patients with unresectable PM with MSI had worse survival compared to MSS PM [53]. In our study, the subgroup of the CRS-HIPEC patients with mutBRAF and MSI had the best survival with 5-year OS exceeding $50 \%$ and median survival not reached. Our main analysis included only PM cases treated with CRS-HIPEC, whereas Sherman et al. included all $\mathrm{mCRC}$ cases with PM regardless of treatment which might in part explain the difference in results [53]. Our results are supported by a study showing that liver resected $\mathrm{mCRC}$ patients with mutBRAF and MSI have a reduced risk of recurrence [46]. MSI cases are important to diagnose as $2 / 3$ of cases benefit from immune checkpoint inhibitors (ICI) in $\mathrm{mCRC}$ [30]. A future research question will be how to integrate $\mathrm{ICl}$ in resectable $\mathrm{mCRC} M \mathrm{MSI}$ cases, as upfront ICI before radical surgery is promising for CRC Stage II-III with MSI [54].

\section{Limitations}

A limitation to this study is the retrospective cohort study design, but the cohort includes all patients given CRS-HIPEC in Norway during a 11-year time period. Management of these patients has changed by utilising better preoperative staging and a shift towards using more preoperative systemic chemotherapy before CRS-HIPEC,. Patients over 75 years of age are not given CRS-HIPEC in Norway and are therefore not included in this study. The molecular data are partly obtained from the primary tumour and partly from metastatic lesions in the peritoneum. However, RAS and BRAF mutations are early molecular tumour changes, and studies have shown a good correlation between mutational status in primaries compared to metastases, and also within different metastasis in the same patient [55]. Possible heterogeneity cannot be ruled out, little data exist but this seems to be less problematic when using tissue from the primary tumour instead of metastases. MSI analyses were only partly available in cases without mutBRAF and analyses of NRAS is lacking.

\section{Conclusion}

The study involves a large cohort of patients with PM-CRC receiving CRS-HIPEC from the Norwegian National Unit for CRS-HIPEC. A surprisingly high proportion of these patients had mutBRAF (24.7\%). Survival after CRS-HIPEC was similar comparing mut $B R A F$, mutKRAS and double wt. The small subgroup with mutBRAF and MSI had better survival. $\mathrm{mCRC}$ patients with a mutBRAF tumour and only limited peritoneal metastasis should be considered for CRS-HIPEC.

\section{DATA AVAILABILITY}

The datasets generated during this study are not publicly available but available from the corresponding author on reasonable request.

\section{REFERENCES}

1. Bray F, Ferlay J, Soerjomataram I, Siegel RL, Torre LA, Jemal A. Global cancer statistics 2018: GLOBOCAN estimates of incidence and mortality worldwide for 36 cancers in 185 countries. CA: A Cancer J Clin. 2018;68:394-424.

2. Manfredi S, Lepage C, Hatem C, Coatmeur O, Faivre J, Bouvier AM. Epidemiology and management of liver metastases from colorectal cancer. Ann Surg. 2006;244:254-9.

3. Thomassen I, van Gestel YR, Lemmens VE, de Hingh IH. Incidence, prognosis, and treatment options for patients with synchronous peritoneal carcinomatosis and liver metastases from colorectal origin. Dis Colon Rectum. 2013;56:1373-80.

4. McCormack PM, Burt ME, Bains MS, Martini N, Rusch VW, Ginsberg RJ. Lung resection for colorectal metastases. 10-year results. Arch Surg. 1992;127:1403-6.

5. van Gestel YR, de Hingh IH, van Herk-Sukel MP, van Erning FN, Beerepoot LV, Wijsman $\mathrm{JH}$, et al. Patterns of metachronous metastases after curative treatment of colorectal cancer. Cancer Epidemiol. 2014;38:448-54.

6. Franko J, Shi Q, Meyers JP, Maughan TS, Adams RA, Seymour MT, et al. Prognosis of patients with peritoneal metastatic colorectal cancer given systemic therapy: an analysis of individual patient data from prospective randomised trials from the Analysis and Research in Cancers of the Digestive System (ARCAD) database. Lancet Oncol. 2016;17:1709-19.

7. Kopetz S, Chang GJ, Overman MJ, Eng C, Sargent DJ, Larson DW, et al. Improved survival in metastatic colorectal cancer is associated with adoption of hepatic resection and improved chemotherapy. J Clin Oncol. 2009;27:3677-83.

8. Tveit KM, Guren T, Glimelius B, Pfeiffer P, Sorbye H, Pyrhonen S, et al. Phase III trial of cetuximab with continuous or intermittent fluorouracil, leucovorin, and oxaliplatin (Nordic FLOX) versus FLOX alone in first-line treatment of metastatic colorectal cancer: the NORDIC-VII study. J Clin Oncol. 2012;30:1755-62.

9. Sorbye H, Cvancarova M, Qvortrup C, Pfeiffer P, Glimelius B. Age-dependent improvement in median and long-term survival in unselected population-based Nordic registries of patients with synchronous metastatic colorectal cancer. Ann Oncol. 2013;24:2354-60.

10. Abdel-Rahman O. A real-world, population-based study of the outcomes of patients with metastatic colorectal cancer to the peritoneum treated with or without cytoreductive surgery. Int J Colorectal Dis. 2020;35:719-25.

11. Verwaal VJ, Bruin S, Boot H, van Slooten G, van Tinteren H. 8-year follow-up of randomized trial: cytoreduction and hyperthermic intraperitoneal chemotherapy versus systemic chemotherapy in patients with peritoneal carcinomatosis of colorectal cancer. Ann Surg Oncol. 2008;15:2426-32.

12. Elias D, Lefevre JH, Chevalier J, Brouquet A, Marchal F, Classe JM, et al. Complete cytoreductive surgery plus intraperitoneal chemohyperthermia with oxaliplatin for peritoneal carcinomatosis of colorectal origin. J Clin Oncol. 2009;27:681-5.

13. Franko J, Ibrahim Z, Gusani NJ, Holtzman MP, Bartlett DL, Zeh HJ 3rd. Cytoreductive surgery and hyperthermic intraperitoneal chemoperfusion versus systemic chemotherapy alone for colorectal peritoneal carcinomatosis. Cancer. 2010;116:3756-62.

14. Mahteme H, Hansson J, Berglund A, Pahlman L, Glimelius B, Nygren P, et al. Improved survival in patients with peritoneal metastases from colorectal cancer: a preliminary study. Br J Cancer. 2004;90:403-7. 
15. Mirnezami R, Mehta AM, Chandrakumaran K, Cecil T, Moran BJ, Carr N, et al. Cytoreductive surgery in combination with hyperthermic intraperitoneal chemotherapy improves survival in patients with colorectal peritoneal metastases compared with systemic chemotherapy alone. Br J Cancer. 2014;111:1500-8.

16. Froysnes IS, Larsen SG, Spasojevic M, Dueland S, Flatmark K. Complete cytoreductive surgery and hyperthermic intraperitoneal chemotherapy for colorectal peritoneal metastasis in Norway: prognostic factors and oncologic outcome in a national patient cohort. J Surg Oncol. 2016;114:222-7.

17. Franko J, Shi Q, Goldman CD, Pockaj BA, Nelson GD, Goldberg RM, et al. Treatment of colorectal peritoneal carcinomatosis with systemic chemotherapy: a pooled analysis of north central cancer treatment group phase III trials N9741 and N9841. J Clin Oncol. 2012;30:263-7.

18. Brucher BL, Piso P, Verwaal V, Esquivel J, Derraco M, Yonemura Y, et al. Peritoneal carcinomatosis: cytoreductive surgery and HIPEC-overview and basics. Cancer Investig. 2012;30:209-24.

19. Passot G, Kim BJ, Glehen O, Mehran RJ, Kopetz SE, Goere D, et al. Impact of RAS mutations in metastatic colorectal cancer after potentially curative resection: does site of metastases matter? Ann Surg Oncol. 2018;25:179-87.

20. Renaud S, Romain B, Falcoz PE, Olland A, Santelmo N, Brigand C, et al. KRAS and BRAF mutations are prognostic biomarkers in patients undergoing lung metastasectomy of colorectal cancer. Br J Cancer. 2015;112:720-8.

21. Sorbye $H$, Dragomir A, Sundstrom $M$, Pfeiffer $P$, Thunberg $U$, Bergfors $M$, et al. High BRAF mutation frequency and marked survival differences in subgroups according to KRAS/BRAF mutation status and tumor tissue availability in a prospective population-based metastatic colorectal cancer cohort. PLoS ONE. 2015;10:e0131046.

22. Schneider MA, Eden J, Pache B, Laminger F, Lopez-Lopez V, Steffen T, et al. Mutations of RAS/RAF proto-oncogenes impair survival after cytoreductive surgery and HIPEC for peritoneal metastasis of colorectal origin. Ann Surg. 2018;268:845-53.

23. Yaeger R, Cercek A, Chou JF, Sylvester BE, Kemeny NE, Hechtman JF, et al. BRAF mutation predicts for poor outcomes after metastasectomy in patients with metastatic colorectal cancer. Cancer. 2014;120:2316-24.

24. Margonis GA, Buettner S, Andreatos N, Kim Y, Wagner D, Sasaki K, et al. Association of BRAF mutations with survival and recurrence in surgically treated patients with metastatic colorectal liver cancer. JAMA Surg. 2018;153:e180996.

25. Kayhanian H, Goode E, Sclafani F, Ang JE, Gerlinger M, Gonzalez de Castro D, et al. Treatment and survival outcome of BRAF-mutated metastatic colorectal cancer: a retrospective matched case-control study. Clin Colorectal Cancer. 2018;17: e69-e76.

26. Gagnière J, Dupré A, Gholami SS, Pezet D, Boerner T, Gönen M, et al. Is hepatectomy justified for BRAF mutant colorectal liver metastases?: a multiinstitutional analysis of 1497 patients. Ann Surg. 2020;271:147-54.

27. Koopman M, Kortman GA, Mekenkamp L, Ligtenberg MJ, Hoogerbrugge N, Antonini NF, et al. Deficient mismatch repair system in patients with sporadic advanced colorectal cancer. Br J Cancer. 2009;100:266-73.

28. Venderbosch S, Nagtegaal ID, Maughan TS, Smith CG, Cheadle JP, Fisher D, et al. Mismatch repair status and BRAF mutation status in metastatic colorectal cancer patients: a pooled analysis of the CAIRO, CAIRO2, COIN, and FOCUS studies. Clin Cancer Res. 2014;20:5322-30.

29. Aasebo KO, Dragomir A, Sundstrom M, Mezheyeuski A, Edqvist PH, Eide GE, et al. Consequences of a high incidence of microsatellite instability and BRAF-mutated tumors: a population-based cohort of metastatic colorectal cancer patients. Cancer Med. 2019;8:3623-35

30. Overman $M J$, McDermott $R$, Leach JL, Lonardi $S$, Lenz $H J$, Morse $M A$, et al. Nivolumab in patients with metastatic DNA mismatch repair-deficient or microsatellite instability-high colorectal cancer (CheckMate 142): an open-label, multicentre, phase 2 study. Lancet Oncol. 2017;18:1182-91.

31. Sinicrope FA. Lynch syndrome-associated colorectal cancer. The. N. Engl J Med. 2018;379:764-73.

32. Graf W, Cashin PH, Ghanipour L, Enblad M, Botling J, Terman A, et al. Prognostic impact of BRAF and KRAS mutation in patients with colorectal and appendiceal peritoneal metastases scheduled for CRS and HIPEC. Ann Surg Oncol. 2020;27:293-300.

33. Benoit L, Cheynel N, Ortega-Deballon P, Giacomo GD, Chauffert B, Rat P. Closed hyperthermic intraperitoneal chemotherapy with open abdomen: a novel technique to reduce exposure of the surgical team to chemotherapy drugs. Ann Surg Oncol. 2008;15:542-6.

34. Strasberg SM, Linehan DC, Hawkins WG. The accordion severity grading system of surgical complications. Ann Surg. 2009;250:177-86.

35. Guren TK, Thomsen M, Kure EH, Sorbye H, Glimelius B, Pfeiffer $P$, et al. Cetuximab in treatment of metastatic colorectal cancer: final survival analyses and extended RAS data from the NORDIC-VII study. Br J Cancer. 2017;116:1271-8.

36. Yang $\mathrm{H}$, Wang K. Genomic variant annotation and prioritization with ANNOVAR and wANNOVAR. Nat Protoc. 2015;10:1556-66.
37. Tol J, Nagtegaal ID, Punt CJ. BRAF mutation in metastatic colorectal cancer. N. Engl J Med. 2009;361:98-9.

38. Lipsyc M, Yaeger R. Impact of somatic mutations on patterns of metastasis in colorectal cancer. J Gastrointest Oncol. 2015;6:645-9.

39. Brudvik KW, Jones RP, Giuliante F, Shindoh J, Passot G, Chung MH, et al. RAS mutation clinical risk score to predict survival after resection of colorectal liver metastases. Ann Surg. 2019;269:120-6.

40. Schweiger T, Liebmann-Reindl S, Glueck O, Starlinger $\mathrm{P}$, Laengle J, Birner $\mathrm{P}$, et al. Mutational profile of colorectal cancer lung metastases and paired primary tumors by targeted next generation sequencing: implications on clinical outcome after surgery. J Thorac Dis. 2018;10:6147-57.

41. Ubink I, van Eden WJ, Snaebjornsson P, Kok NFM, van Kuik J, van Grevenstein WMU, et al. Histopathological and molecular classification of colorectal cancer and corresponding peritoneal metastases. Br J Surg. 2018;105:e204-e11.

42. Chu DZ, Lang NP, Thompson C, Osteen PK, Westbrook KC. Peritoneal carcinomatosis in nongynecologic malignancy. A prospective study of prognostic factors. Cancer. 1989;63:364-7.

43. Lochhead $P$, Kuchiba $A$, Imamura $Y$, Liao $X$, Yamauchi $M$, Nishihara $R$, et al. Microsatellite instability and BRAF mutation testing in colorectal cancer prognostication. J Natl Cancer Inst. 2013;105:1151-6.

44. Brunsell TH, Sveen A, Bjørnbeth BA, Røsok BI, Danielsen SA, Brudvik KW, et al. High concordance and negative prognostic impact of RAS/BRAF/PIK3CA mutations in multiple resected colorectal liver metastases. Clin Colorectal Cancer. 2020;19:e26-e47.

45. Schirripa M, Bergamo F, Cremolini C, Casagrande M, Lonardi S, Aprile G, et al. BRAF and RAS mutations as prognostic factors in metastatic colorectal cancer patients undergoing liver resection. Br J Cancer. 2015;112:1921-8.

46. Bachet JB, Moreno-Lopez N, Vigano L, Marchese U, Gelli M, Raoux L, et al. BRAF mutation is not associated with an increased risk of recurrence in patients undergoing resection of colorectal liver metastases. Br J Surg. 2019;106:1237-47.

47. Baratti D, Kusamura S, Niger M, Perrone F, Milione M, Cattaneo $L$, et al. Prognostic impact of primary side and RAS/RAF mutations in a surgical series of colorectal cancer with peritoneal metastases. Ann Surg Oncol. 2020;27:98-106.

48. Loupakis F, Intini R, Cremolini C, Orlandi A, Sartore-Bianchi A, Pietrantonio F, et al. A validated prognostic classifier for V600EBRAF-mutated metastatic colorectal cancer: the 'BRAF BeCool' study. Eur J Cancer. 2019;118:121-30.

49. Seligmann JF, Fisher D, Smith CG, Richman SD, Elliott F, Brown S, et al. Investigating the poor outcomes of BRAF-mutant advanced colorectal cancer: analysis from 2530 patients in randomised clinical trials. Ann Oncol. 2017;28:562-8.

50. Taieb J, Le Malicot K, Shi Q, Penault-Llorca F, Bouché O, Tabernero J, et al. Prognostic value of BRAF and KRAS mutations in MSI and MSS stage III colon cancer. J Natl Cancer Inst. 2017;109:djw272.

51. Taieb J, Zaanan A, Le Malicot K, Julié C, Blons $H$, Mineur L, et al. Prognostic effect of BRAF and KRAS mutations in patients with stage III colon cancer treated with leucovorin, fluorouracil, and oxaliplatin with or without cetuximab: a post hoc analysis of the PETACC-8 trial. JAMA Oncol. 2016;2:643-53.

52. Tran B, Kopetz S, Tie J, Gibbs $P$, Jiang ZQ, Lieu $C H$, et al. Impact of BRAF mutation and microsatellite instability on the pattern of metastatic spread and prognosis in metastatic colorectal cancer. Cancer. 2011;117:4623-32.

53. Sherman SK, Schuitevoerder D, Chan CHF, Turaga KK. Metastatic colorectal cancers with mismatch repair deficiency result in worse survival regardless of peritoneal metastases. Ann Surg Oncol. 2020;27:5074-83.

54. Fujiyoshi K, Yamamoto G, Takenoya T, Takahashi A, Arai Y, Yamada M, et al. Metastatic pattern of stage IV colorectal cancer with high-frequency microsatellite instability as a prognostic factor. Anticancer Res. 2017:37:239-47.

55. Østrup O, Dagenborg VJ, Rødland EA, Skarpeteig V, Silwal-Pandit L, Grzyb K, et al. Molecular signatures reflecting microenvironmental metabolism and chemotherapy-induced immunogenic cell death in colorectal liver metastases. Oncotarget. 2017:8:76290-304.

\section{ACKNOWLEDGEMENTS}

We thank hospitals in Norway for their collaboration in referring patients to the National Treatment Unit for CRS-HIPEC in Norway located at Norwegian Radium Hospital, part of Oslo University Hospital. We also thank Shakithiya Thiyagarajahm, Stine Beate Hansen, Michelle Tune, Susan Shahzidi and Liv Inger Håseth for extracting DNA and performing the additional analyses at Oslo University Hospital. Data from this study is partly presented in poster \#3565, ASCO 2019.

\section{AUTHOR CONTRIBUTIONS}

SL has performed all parts of the study from treatment to database work, idea, statistical analyses, preparing of figures and tables and writing the manuscript. He has had full access to the data in the study and has the final responsibility for the 
decision to submit for publication. All authors have played an important role in interpreting the results, revised the manuscript, agreed to the work and approved the final version. In addition MG, SD and KF have in addition taken part in the treatment and handling of the patients. SS, EH, AT, HS and MLI have in addition collected molecular analysis in the study. VJD has in addition performed statistical analyses and preparing of figures. HS has in addition to parts specified over, also contributed widely with the idea and preparing of the manuscript.

\section{FUNDING}

Grant support: Erika Stekels Foundation, Norwegian Cancer Society, Oslo, Norway for molecular analysis, otherwise none.

\section{COMPETING INTERESTS}

The authors declare no competing interests.

\section{ETHICS APPROVAL AND CONSENT TO PARTICIPATE}

The study was approved by the Norwegian Ethics Committee (s-07160b), SouthEastern Norway Regional Health Authority, Hamar, Norway and written informed consent was obtained from the patients. Data are from our institutions peritoneal metastasis MedinSight database, Oslo University Hospital, Oslo, Norway. Base owner SL is Head of Oncological Surgery, The Norwegian Radium Hospital, and Chair of the National Service for CRS-HIPEC in Norway. The study is performed in accordance with the Declaration of Helsinki.

\section{CONSENT TO PUBLISH}

No individual person's data is included.

\section{ADDITIONAL INFORMATION}

Supplementary information The online version contains supplementary material available at https://doi.org/10.1038/s41416-021-01620-6.

Correspondence and requests for materials should be addressed to S. G. Larsen.

Reprints and permission information is available at http://www.nature.com/ reprints

Publisher's note Springer Nature remains neutral with regard to jurisdictional claims in published maps and institutional affiliations.

Open AccessThis article is licensed under a Creative Commons Attribution 4.0 International License, which permits use, sharing, adaptation, distribution and reproduction in any medium or format, as long as you give appropriate credit to the original author(s) and the source, provide a link to the Creative Commons licence, and indicate if changes were made. The images or other third party material in this article are included in the article's Creative Commons licence, unless indicated otherwise in a credit line to the material. If material is not included in the article's Creative Commons licence and your intended use is not permitted by statutory regulation or exceeds the permitted use, you will need to obtain permission directly from the copyright holder. To view a copy of this licence, visit http://creativecommons. org/licenses/by/4.0/.

(C) The Author(s) 2021 